\title{
Intermédialités
}

Histoire et théorie des arts, des lettres et des techniques

Intermediality

History and Theory of the Arts, Literature and Technologies

\section{The Sounds of Disappearance}

\section{David Guimond}

Numéro 10, automne 2007

Disparaître

Disappearing

URI : https://id.erudit.org/iderudit/1005556ar

DOI : https://doi.org/10.7202/1005556ar

Aller au sommaire du numéro

Éditeur(s)

Centre de recherche sur l'intermédialité

ISSN

1705-8546 (imprimé)

1920-3136 (numérique)

Découvrir la revue

Citer cet article

Guimond, D. (2007). The Sounds of Disappearance. Intermédialités /

Intermediality, (10), 115-130. https://doi.org/10.7202/1005556ar

\section{Résumé de l'article}

L'observation des micro-opérations des propriétés physiques du son permet de dépasser la temporalité qui a jusqu'ici servi à en distinguer l'apparition et la disparition en ce qui touche la " venue » et l'« estompement ». Caractérisé par une vive activité hétérogène au plan physique, le son est capable d'engendrer des registres simultanés d'apparition et de disparition dans lesquels ceux-ci ne peuvent ni s'exclure mutuellement ni se distinguer clairement, ce qui devrait nous inciter à éviter la simple dichotomie temporelle. Il semble plutôt que la puissance du son tienne dans l'entremêlement de l'apparition et de la disparition, simultanément, dans la création d'événements sonores selon un sujet à l'écoute. 


\title{
The Sounds of Disappearance
}

\author{
David GuImond
}

THE PHYSICALITY AND TEMPORALITY OF APPEARANCE

AND DISAPPEARANCE IN THE DISINTEGRATION LOOPS

In 2001, William Basinski, a sonic artist who had experimented with tape loops for nearly 30 years, rediscovered one particular set of tapes that he had recorded in 1982 but which he had never used or altered on his own. He immediately went about transferring the reel-to-reel tapes to digital format, but time had ravaged the integrity of the actual magnetic strips. As the tapes began to roll, the iron oxide covering the tapes began to disintegrate, small flecks of it falling off as they were scraped away by the reeler head, leaving random bare spots on the tape, while at the same time the fragile tapes began to stretch past the point of their normal taut integrity, both processes warping the structure and melody and sound of each loop as they were being recorded digitally. He eventually released The Disintegration Loops as four disks comprising nine unique tracks, each one ranging anywhere from 10 minutes to well over an hour, with a running time of just over 5 hours.

The effect of this unintended alteration on the music is unmistakable. Each track employs one loop throughout the piece-mostly short snatches of brass band, interspersed with shortwave radio static, characterized by a lush string melody and backed by atmospheric arpeggio countermelodies-which, due to the deterioration process, is constantly changing from one repetition to the next. The tracks may begin as thoroughly bold orchestral melodies, their sound crisp and even; however, one begins to hear, sometimes imperceptibly, the tapes literally deteriorate with each turn of the reeler head. Letting silences and static bleed through the cracks, the sound slowly unravels as the loops stumble out from and back into the void, the structure of the original loop changing with every re-emergence, the process slowing down and increasingly warping every subsequent loop's melody and rhythm. The integrity of the individual loops become increasingly problematized as they then start to seep into each other, opening up an "inter-texture-ality" of ever more mutated echoes, staggered silences, and 
misshapen spaces, "somnambulant sounds that drift in and out; sounds that tiptoe around the house like ghosts." The music eventually becomes just a shadow of itself, painfully reduced to intermittent bursts of sound that simultaneously resemble nothing like the opening loop yet are still recognizable, haunted as they are by the hazy ghost of its original structure, but now completely dismantled and warped. Reduced to but a whispered outline of this structure, even these gasping chords, towards the end barely perceptible as the last loop tortuously tries to force itself into appearance only to fade away quietly, disappear along with the whole track, into nothingness, "leaving a stunningly beautiful metaphor-an impression of a world disappearing." In these ways, just like sound, The Disintegration Loops exist through a simple modulation of appearance and disappearance, each loop appearing, vibrating, expending energy, being heard and disappearing.

Common responses by reviewers to this process within the work is that The Disintegration Loops is framed most apparently as a meditation on the nature of time, musing on birth, entropy, decay, and how these reverberate in memory. It is evident that time is crucial to The Disintegration Loops not just as a musical unfolding, the loops, a slow diffusion of waves, coming into being and fading away, but also at the level of the process of disintegration where time has literally bled into and ravaged the tapes. The mediatic disintegration that occurs in chorus with the sonic disintegration speaks to a conception of sound that can never be separated from its simultaneously occurring temporality and materiality. The mediatic materiality of the loops, are folded into time such that its sounds only emerge through them, serving as a not just a marker of passing (linear) time but also the "place" where the materiality of time is crushed, stretched, and warped. In this sense, time and sound, media and materiality, are inextricably bound to the emergence and production of each other such that they are impossible to separate, bridging the divide between metaphorical and material disappearance. More specifically, as with anything in nature, the loops are captured by the remorseless nature of entropy, and so the theme of death looms over and is interwoven in the work, one which also fades in and out of the metaphor/mediatic divide. Tapes that reappear after 20 years are literally disappeared as they are reworked, killed off in their transplant from a slowly dying analog media (and rebirthed again digitally), making their entropic ruin the (un)structured principle by which the loops unfold their metaphorical deaths where, as one reviewer frames it, "transforming a throbbing atmosphere of delicate infancy rememberings into a flesh-tearing

1. Audra Shroeder, New Times - Broward Palm Beach, 6 May, 2004, 2. Retrieved on 15 July 2004 from www.newtimesbpb.com/issues/2004-05-06/shortcuts3.html.

2. Alexander Provan, June 30, 2004, 33 Retrieved on July 15, 2004 from www. dustedmagazine.com/reviews/1444. 
interruption," they all die very individual deaths. ${ }^{3}$ This is the law of entropy insinuated into the media's materiality becoming translated metaphorically into music, in which any and every sound struggles to emerge, becomes less frequent and more mutated, the spaces between them becoming longer and more drawn out. Because of this, reviewers hear in the piece a sense of mourning and loss haunting the music as it unfolds its own life, the loops gently slipping away, "the sound of life as it decays and dies before our ears. And like all living things, these sounds struggle and claw for life with their last, dying breaths." life itself, fade softly into silence. However, despite this somber mood, there is also an undercurrent of hope and renewal, Basinski simultaneously engaging the theme of birth and rebirth throughout, himself acknowledging this in his liner notes: "Life and death were being recorded here as a whole: death as simply a part of life: a cosmic change, a transformation." F From the musical loops being materially "born" in 1982 and "reborn" in their rediscovery in 2001, to the musical births of each new loop as it assumes a new shape, sound and structure after each individual disintegration, the loops have been read as mediatically and metaphorically contemplating not only the moment of creation, but also regeneration. One reviewer writes that he imagines that "life within the womb might sound something akin to [this]," while another that "[the music] brings to my mind the effort of [...] giving birth to [Basinski's] creature: repetition and the ongoing pulverizing of sound resemble the extreme force of life desperately wanting to overcome."7 And overcome it does, as each death is an eventual rebirth, through the repetition of the loops and the cyclic nature of the work (where the first track and the last track employ the same loop $^{8}$ ) which as a whole becomes a circular epic, "the soundtrack of unbelievable destruction and new possibilities."

As elusive and haunting as the loops themselves, memory becomes the ethereal substance that connects these various themes, with Basinski both mourning

3. Massimo Ricci, Touching Extremes, no date. Retrieved on July 15, 2004 from www.spazioinwind.libero.it/extremes/touchingAD.htm (website deactivated).

4. Michael Heumann, no date, $\$ 6$. Retrieved on 15 July 2004 from www.hauntedink. com/25/basinski-disintegration.html

5. William Basinski, Liner Notes in The Disintegration Loops, 2002 (2062 Music).

6. Joe Tangari, 9 April 2004, \$3. Retrieved on July 15, 2004 from www.pichforkmedia. com/record_reviews/b/basinski_william/disintegration-loops.shtm

7. Massimo Ricci. Touching Extremes.

8. Basinski had recorded two copies on tape of this particular loop so that, while the first and last track employ the same loop, their sound, through different degrees of disintegration, is warped differently.

9. Just Add Noise, no date, $\$ 4$. Retrieved on July 15, 2004 from www.justaddnoise. com/reviews/b/basinski_disintegration_I.htm 
and accepting the loss of his own memories: "tied up in these melodies were my youth, my paradise lost, the American pastoral landscape, all dying gently, gracefully, beautifully." $1 \circ$ Time may have decayed the ideals the loops once represented, but the loops become, at one level, the preservation of those memories-musically in its echoes, structurally in its cyclic nature-that were once youthful and fresh. This is where some reviewers have found the humanity of the work so deeply felt, where, as transient beings, we feel "the implicit, human need to remember, to hold on to things going away forever," ${ }^{11}$ and in this sense, the music becomes "a long reflection on what's become of us after so many beautiful moments of our life." ${ }^{2}$ However, the untrustworthy instability of memory prevents this, such that at another level, the loops also represent the impossibility of memory, the difficulty of remembering the moments of our life as how they happened, where time, like the disintegration process, warps and "disappears" everything. Ultimately and poignantly, the tapes and the music are the sound of fading and shifting memories, losing microscopic fragments with every loop until the tiniest molecule of sound is gone, "until the last screen veiling the music's moment of extinction is lowered, upon which vague memories of people and times long forgotten are projected, little shadows dancing on.,"13

\section{THE HETEROGENEITY OF APPEARANCE AND DISAPPEARANCE IN THE VIBRATORY FABRIC OF SOUND}

Sound discernably follows a temporal trajectory from appearance to disappearance, emerging into, existing and then fading from first a medium and then perception. However, once opened up beyond the temporality that functions as the most apparent modulation of its appearance/disappearance and analyzed at the level of its physical micro-operations, sound displays an ability to engender simultaneous registers of appearance and disappearance on numerous levels. Imbued with both materiality and ephemerality at once, sound's appearances and disappearance come to be characterized by frenetic levels of heterogeneous activity which invite us to eschew their simple dichotomization by constantly pushing forth and allowing to dissipate different meanings and histories of sound. In other words, examining sounds' physical properties shows us that its appearance and disappearance are not mutually exclusive and that they can neither be clearly separated nor properly distinguished as a here/not-here or a now/not-now, as easily

10. William Basinski, Liner Notes in The Disintegration Loops.

11. Logoplasm. Retrieved on July 15, 2004 from www.logoplasm.org/sagita/diffusion (website deactivated).

12. Massimo Ricci.

13. Logoplasm. 
done if only considered through its temporality. Rather, the power of sound functions specifically because it forever intertwines its appearances and disappearances simultaneously in the creation of the sonic event with the listening subject.

To begin considering sound's heterogeneity, we should look at the context in which a sound unfolds. Compared to how light waves characterize vision, the higher wavelength and mechanical nature of sound waves dictate that their propagation is more diffuse and slow as they rely on the qualities and large-scale stress and relaxation of whatever elastic medium through which they are transmitted, accounting for our heightened sense of the movement, flux and contingency of sound. As such, sound waves are not only necessarily bound to a context in order to propagate but are also influenced by the general configuration of that environment. This inseparability, as the wave's unfolding travels has it changed by each interaction with the environment as it reflects off and is absorbed by all objects, means that "a sound wave arriving at the ear is analogous of the current state of the physical environment."14 Therefore, the compositional makeup of a sound wave is one in which the structural simplicity or purity of its qualities become increasingly complicated or tainted through its interaction with all of the objects (including other sonic objects) in the path of its diffusion in the environment; a complication which simultaneously opens up the multiplicities of sound's appearance and disappearance. As it emanates out, the energy that comprises a sound wave simultaneously disappears exactly as it is expended and appears in the medium, while at the same time the measure of the physical qualities (frequency and amplitude) that comprise the (original) wave are shifting constantly through various appearances and disappearances: they disappear as the wave's energy is constantly transferred and appears in other objects and media which are in the path of its diffusion; they are altered as the wave reflects off everything in its path; and they are supplemented by the appearance of the qualities of other sound waves that are either already present in the medium.

These shifting appearances and disappearances also hold true for a sound wave's interaction with other sonic objects, which can be either existing sound waves (already traveling through the environment) or those that have emerged from being forced into vibration by the original sound wave. For example, in the phenomena of resonance and forced vibration, ${ }^{15}$ the pressure created in the

14. Barry Truax, Acoustic Communication, Westport, CN, Ablex Publishing, 2001, p. 17.

15. Resonance occurs when one vibrating object elicits vibration from another because they share the same natural frequency and are connected by surrounding particles. However, even if objects do not share the same natural frequency, the nature of vibrations is such that the pressure created in the medium by a vibrating object will force any adjoining or interconnected object into vibrational motion, and this phenomenon is known as forced vibration. 
medium by a vibrating object will elicit some degree of vibration from any object with which it is interconnected (through particles of the medium). The object forced into vibration will emanate a different vibratory pattern, but one which is forced to interact with and become incorporated with the original sound wave. In this interaction, parts of the original sound wave disappear in favor of the appearance of qualities emanating from the object forced into vibration, producing a new sound wave that is structurally different from the one that emanated from the original vibrating object. For example, in attempts to produce a truly pure tone in acoustic laboratory tests under controlled conditions "it is doubtful whether by the time the tone has reached our ears it has not been corrupted by resonances picked up on the way." ${ }^{16}$ Even a solitary sine wave, describing one element of a sound, "once given life in a room, will excite its harmonics in sympathetic bodies." ${ }^{\prime 7}$ Because of these interactions, a sound wave is never fixed: as some qualities emerge and others dissipate in a constant process of disappearance and appearance in the diffusion of a sound wave through the environment, constantly changing its structure and texture as it becomes enmeshed with and comes apart from other media and vibrations, ultimately, at the point of emergence, a sound represents "the fused sum of all the vibrations to reach this point in time and space." (SI, p. 14)

The sum of this interaction between a sound wave and the environment creates a complex, vibratory fabric composed of an infinite, multiplicity of sound waves in which we are forever immersed in both its materiality (as a substance that moves through us and rubs against us) and its immaterality (as a substance that cannot be held, contained or seen). Underlying the sound that we hear is this vibratory fabric which, when disturbed, tenses and relaxes to produce a sound, but when "at rest" is still comprised of an endless ocean of different sound waves, overlapping and layered, each one comprised of various frequencies and amplitudes, struggling violently against each other in such a dense flux that it is impossible to demarcate when the qualities that comprise a sound appear and when they have disappeared. While the sound we eventually hear requires specific thresholds to be reached, even when not coming to appearance in the form of an audible sound, all of these qualities are still forever there, surrounding and penetrating our body, waiting to unfold in a sonic event:

16. Henry Cowell, "The Joys of Noise," in Cristoph Cox and Daniel Warner (eds.), Audio Culture: Readings in Modern Music, New York, Continuum, 2004, p. 23.

17. Aden Evens, Sound Ideas: Music, Machines and Experience, Minneapolis, University of Minnesota Press, 2005, p. 47. Henceforth, references to this text will be indicated by the initials "SI," followed by the page number, and placed between parentheses in the body of the text. 
Every vibration, every sound, hangs in the air, in the room, in bodies. Sounds spread out, they become less and less contracted, they fuse, but still they remain, their energy of vibration moving the air and the walls in the room, making a noise that still tickles the strings of a violin playing weeks later. Every sound masks an entire history of sound, a cacophony of silence. (SI, p. 14)

The physical properties of how a sound wave interacts with the vibratory fabric are such that what is eventually heard as one sound contains within it the continually supplemented appearance of the histories of other potential sounds to itself, but is also defined by its own histories, and by other potential sounds that have disappeared and decayed along the way. Through this heterogeneity, this "entire history of sound" speaks to how an individual sound, even before coming to perception, is in a constant state of fluid and overlapping appearances and disappearances, troubling the ability to determine how and when these occur.

And yet, even if we reduce our scope from the sonic fabric to an individual sound wave, it remains capable of producing multiplicities through its heterogeneity. Consider one sound wave. While what we eventually hear as one sound appears uniform, the compositional makeup of a sound wave is not only a complex of heterogeneous sine waves describing its various physical characteristics, but its compression at a particular frequency is not a uniform process. A compression acts as a centre of power for sound, bringing together a vast array of different sine waves describing various parts and qualities of the sound wave and then imposing on this chaotic irregularity a uniformity such that, in sound's sounding, the repeatable iteration of these high pressure areas will appear to be a stable expression that falls within the range of human hearing. However, when attempting to impose this uniformity, the compression process must rely on rarefactions, the area where the physicality of sound's energy has dispersed; in this way, disappearances insinuate themselves into and help comprise the quality of the eventually-heard sound on numerous levels. At the first level, in drawing sonic elements together, each compression pulls into itself and thus implicates some of the properties of the compressions and rarefactions that come before it and, as such, it becomes implicated in those that come after it; thus, some of the sonic properties of rarefactions become drawn into compressions. It is this operation of a compression's "overflow" which allows the ear to hear, for example, individual notes that comprise a sound both as individually distinct and as part of a movement. At another level, while some of the properties of unused vibrations are never drawn into a compression and remain in the low-density area, they can still influence the hearing process because a sounding needs rarefactions to present itself as a variation in pressure for the ear to vibrate. In these ways, rarefactions subvert the uniformity and power of compression by implicating the properties and histories of a series of not-sounds and disappearances into what 
will eventually be heard in the sonic event, now to be underscored by shifting patterns of loss.

By gathering heterogeneous sonic elements, by opening itself to rarefactions, by containing elements that came before it, and by opening itself to have its own elements drawn into compressions that come after it, the compressiondespite what may be the uniformity and smoothness of a heard sound-does not "gather into a uniform whole but collects heterogeneously, making an assymetrical, weighted duration that spills over its edges." (sI, p. 40) As such, each compression that comprises one sound is itself both related to and unique from other compressions. The continual appearance of certain qualities of the sound wave marks it as a part of a movement that as it has been chosen to be made uniform forms its relationship to other compressions. It is at the same time unique because each compression is differentially composed of various degrees of disappearance, dependent as it is on the qualities of the rarefactions it is related to. Thus, even at the subatomic level, when the physical elements of a sound wave are compressed into the character of a sound (i.e. those qualities which make the various histories of a wave's appearances appear as a sound appears), each compression includes the histories of its own various disappearances, ones that are eventually heard in the sound's quality. That which appears as sound is heard this way because the compression process makes other qualities disappear from the heard sound, but in such a way that their disappearance still affects the quality of the heard sound.

These peculiar operations again point to the constant production of heterogeneity at various levels of sound, in its movement, interaction, composition, compression and continuous corruption and reconstitution, all folded into the vibratory fabric that surrounds the emergence of each heard sound. As soon as an object is set into vibration, even if a sound's sounding may appear to us in perception as texturally smooth or constant or uniform, once dissected it is exposed to be a chaotic composition of its multiple histories of appearance and disappearance. The various levels of heterogeneity in sound detail an excessive multiplicity which refuses to let its appearances and disappearances exist as a simple dichotomization, of a fading in and then fading out; while "the whole of sound is contracted each time, as noise gathers the entire history of sound into each point," (SI, p. 18) when a sound appears, part of this history necessarily includes its very own not-sounds that have disappeared.

\section{APPEARANCE, DISAPPEARANCE AND THE LISTENING SUBJECT}

When we come to the level of the heard sound, while the vibratory fabric of sound may constantly surround us, it is only in the perception of sound that it inscribes the body its heterogeneous histories of appearances and disappearances, 
the continual perception itself a continual inscription on the body. Just as sound requires a disappearance of sonic elements to be perceived as a heard sound as it appears to the body, its disappearance from the body as its energy fades is its simultaneous appearance in the body as it marks the subject with its histories through the process of memory in the co-constitution of the sonic event with the listening subject. Further, this process is perpetually being reconstituted in such a way that this appearance is never stable, never complete, thus leaving its resolution always open to renegotiation by the listening subject.

By implicating heterogeneous sonic elements that come before it and after it, the compression becomes "a weighted hearing that is open to the next moment precisely because it retains the last one," (SI, p. 42) spilling over the edges of its own meaning and resolution. This asymmetry ensures that it neither creates a homogeneous block of time-sound nor resolves itself into a specific sound. Rather, it pushes forth a series of sonic singularities, specific but not specified moments in the substance of sound that bend towards the ear, "implicating worlds of forces not yet unleashed but whose reserve powers the music, drives it along," (SI, p. 17) each one containing its various pasts and potential futures, each one necessarily open to further articulation by the hearing subject, ensuring that whatever the heard sound will eventually be is only implied rather than resolved in its compression:

What is implicated is not an obscure version of the music yet to come, not a specific direction for the music to take, nor even a bunch of possible directions. Implication does not gather within it the specific vibrations that will be expressed as variations in air pressure. Rather, the implicated is obscure by its nature, incorporating not so much the clarity of sound waves as the singularity of events, historical events, musical events, masking within it affect apart from object and percept without subject. The implicated contracts noise, an entire history of sound, but the contracted events, percepts and affects are still inarticulate, too relaxed to be clear. They are singular but not specific. Unlike expectation, implication does not specify the resolution, the harmonic possibilities, the melodic continuation, though it does establish the realm from which the possibilities for these specifics can be drawn. In contracting noise to the point of clarity, implication brings close to the surface some of the depth of noise, powering the music while focusing the next contraction, the next repetition. (SI, p. 20)

In other words, a compression implicates the sonic singularities of the past, "the history of sound", and pushes these towards a future without specifying a direction or resolution for the sound, but rather bringing both of these "close to the surface" of hearing. It is the hearing subject who must then actively compress a heterogeneity of "inarticulate" heterogeneous compressions into its perception, 
resolving all of the various physicalities of sound into a heard sound by bringing them from "the point of clarity" into a uniform and constant texture. In this way, the body is at the centre of this process, crushing time and opening the implied in sound, just as it is sound itself that opens the body to inscription.

Transpiring in the body, the locus of this inscription by sound is in memory specifically, where even at the physical level of sound's qualities, the body must remember:

To hear pitch and timbre, one must remember the last few vibrations of the air.

These few milliseconds are compressed, drawn together, their interval difference extinguished, covered, or crossed by the compression. One does not hear a variation of air pressure, a difference over time, but only the effect of this difference, the steady quality of pitch and timbre. (SI, p. 41)

But because every compression and sound wave are composed of histories of vibrational appearances and disappearances, when a sound is contracted into perception it indelibly marks the body with these histories, being inscribed into us through the need to remember its sonic qualities. What first appeared to the body, as sound waves approach us through our immersion in the sonic fabric that surrounds us, these histories of sound now appear in the body in perception. Part of what is implicated, that which will forever be pushed forth as sonic singularities to be resolved by the listening subject, include sound's own histories of appearance and disappearance. In this way, how sound appears to us in its materiality partially shapes our subjectivity through its disappearance. I say partially because we cannot discount the role of the listener in shaping the sonic experience; our immersion in sound should not only be looked at in terms of how its force can unidirectionally constitute the sonic event and thus the subject in a total way. While we can say that there is a sonic envelopment that brings forth sonic singularities and that there will always be something in sound that seems beyond listeners' control, auditory perception is much more being subjected to these, and powerless, because the final constitution and reconstitution of the sonic event can only be co-determined with the listening subject. Simply, in the sonic event, while the "sound must be bent to invite the listener to share in its meaning," the listener must, as Evens insists, "bend [their] hearing just so, in concert with the sound [he or she] hears." (SI, p. 28) Within the sound, this "bending" is evident in how each compression is a sonic singularity that does not specify its own resolution but establishes a field of possibilities from which specific meanings can be drawn, simply bringing sound to the point of clarity. It is at this point that the active listener draws from this "realm" of possibilities their own meaning of the sound and helps constitutes the sonic event, the resolution of which transpires only with the listening subject "bending" his or her ear to the 
sound. As we have already discussed, within this event and the actual physical properties of a sound are histories of appearance and disappearance in every compression, in every wave and thus in every contraction into perception. So in the process of "bending" towards the sound, its physical properties mark the body with these histories through the process of memory, which places together the movement of the weighted durations to make sense of them. But at the same time, the resolution of these possibilities and histories implicit in sound's rhythmic physical qualities occurs in conjunction with the histories of the subject whose listening is bent in an aesthetic of bodily involvement, the body itself taking sound's histories and implicating its own. Therefore, the continual bringing forth of sound's histories and movement is a continual working on the subject who partially resolves the multiple possibilities because of and within his or her own history in the coproduction of the sonic event, such that what a sound "is," is a personal incorporation of histories that cannot be generalized, but whose resolution of the sonic event inevitably makes it a political one because it is a corporeal one. However, the resolution of the sonic event forever remains only partial because sound's perpetual movement constantly re-enacts this process in hearing: "[...] the contraction is always repeated, the implicated always implicates itself again [...] this repetition, this reimplication that moves sound forward, pushing music along," constantly bringing forth the different histories and singularities of sound in its opening up to the future possibilities of resolving the sonic event, as "[...] contracted sound immediately relaxes again, falling back into the noise it rose out of and allowing other contractions to come to the fore." (SI, p. 18) Therefore, just as the heard sound begins its own disappearance from the body, this entire process begins again with new compressions, new waves and new sounds, making the process and its resolution forever unstable, always partial and never complete, and, because of this, bursting with histories that are always open to reconstitution by the hearing subject.

Taking the physical qualities of sound seriously, therefore, "does not mean that we have to give up on the discursive formation of subjectivity," rather, as Revill contends, "one might argue that if, as many cultural theorists believe, we as human beings in society are inevitably pitched into discourse, and if discourse is fundamental to our 'being-in-the-world', then the discursive is embodied in sound, in rhythm, timbre and vibration. ${ }^{18} 8$ Subjectivity spreads out and merges with the sonic histories of appearances and disappearances that are folded into sound waves and the sonic fabric, caught in a constant mutual constitution of self

18. George Revill, "Music and the Politics of Sound: Nationalism, Citizenship, and Auditory Space”, Environment and Planning D: Society and Space, Vol. 18, No. 5, 2000, p. 604 . 
and other as their boundaries dissolve, leading to a sonic fragmentation of the subject who becomes what Connor has referred to as the "phantasmic soft body, held in suspension in the synaesthetic coilings of the ear, between the inside and the outside, the self and the other."19

\section{APPEARANCE AND DISAPPEARANCE IN THE SOUND OF THE DISINTEGRATION LOOPS}

There are many structural similarities between the elements of the vibratory fabric that help sonically constitute subjectivity and the overall form/content/ process of The Disintegration Loops. There is an entire process of action and interaction between tape, tape head, the sound of the original loop, its disintegration and the sounds of the disintegrating loops, such that each track can be considered a vibratory fabric in its own right, characterized by the constant activity of vibrating objects, a temporality necessary for it to unfold and a heterogeneity of the sonic properties of each loop. By constantly pushing forth the disintegrating loops as individual sonic singularities which are structured and characterized by both appearances and disappearances, their unfolding brings these histories into appearance for the listening subject, only to disappear, their repetition marking the body but signaling the continual and mutual re-constitution of the event.

To elaborate, if one imagines the original loop of a track as an object that undergoes vibration, the disintegration as the process which initiates vibration, the actual physical object of the magnetic tape onto which the loops were recorded as a medium akin to the medium necessary for the transmission of sound and the subsequent loops of the track as the resulting sound waves emerging and flowing from the vibrating object, one hears The Disintegration Loops behaving much like the various processes of sound just discussed, as a dense and active flux that complicates modulations of appearance and disappearance by refusing to be fixated in the proliferation of its multiple histories, and refusing to separate the metaphorical from the material. The disintegration of tape translates an evident elasticity of a medium into the sonic stress and relaxation of the loops which changes the sound and structure of each loop and produces areas of sound/compressions and not-sound/rarefactions in the piece, such that the sound of the loops are bound to and inseparable from the context of that medium. Like any vibrating object, once the original loop begins disintegrating, it sets into motion a series of interconnected, vibrating loops which, much like

19. Steven Connor, "Edison's Teeth: Touching Hearing," in Veit Erlmann (ed.), Hearing Cultures, New York, Berg Press, 2004, p. 171. 
sound waves, diffuse slowly outwards from it, each loop disturbing and interacting, reflecting and overlapping, with its nearing neighbor, both being absorbed into or absorbing other loops and transferring this energy throughout the whole track. In this disintegration, the loops have no clear boundaries between them, spreading out in an autonomous spatial and temporal existence away from the original loops, bleeding into and interacting amongst themselves and changing their spatiotemporal vectors and sonic qualities each time. In this way, the loops follow patterns of appearance and disappearance similar to sound waves: both are manifested energy and contingently affected, material and ephemeral. This dynamism ensures that their everchanging properties are characterized by the appearances and disappearances of other loops and waves. Like sound waves and vibrations, listening to the loops, as they vibrate, propagate, dissipate, their energy conserved as they move away from the original loop but expended throughout the piece, is like listening to the vibratory fabric of sound waves that surrounds us. The whole becomes a dynamic environment distributing sound's energy across its medium in a continuous variation of pressure heard in the shifting sounds of each loop, each loop comprised of different and interacting sonic properties, their unfolding comprised of different sounds which at different times emerge, overlap, compete, crash headlong, relax and then dissipate, underscored by a pattern of appearances and disappearances which shape the vibratory fabric in its unfolding. The energy and integrity of the loops eventually become less stable yet still affect the sonic unfolding of the next loop, the stress and relaxation of the tape dancing on the thresholds on sound/not-sound, heard in the struggle for the loops to emerge and remain heard, until this energy expends itself, fading into silence at the end of the track.

We can also extrapolate Evens' ideas on compressions into the action that transpires in The Disintegration Loops. If we reduce our scope and cast the original loop of a track as a uniformly heard sound, the disintegration process can be heard as an opening up of this uniformity and an emerging of all the heterogeneous sonic elements that actually comprise it, constantly changing the sonic properties of the sound. Beneath each loop as a compression of sound and each disintegration as its rarefaction, lies a chaotic and dynamic multiplicity of space, time and sound. Just like every compression, each loop similarly acts as a gathering of heterogeneous sonic properties trying to impose a uniformity in its sounding, but different each time-corrupted as it is by the interpenetrating resonances and forced vibration of the disintegrating loops changing the quality of the heard sound-becoming a "weighted hearing" that simultaneously absorbs the properties of the preceding loop and influences the properties of the next loop. Containing its own past and its own future, these asymmetries allow a 
loop to simultaneously relate to both the preceding and following sound while remaining differentiated in a duration that spills over its edges, as each loop bleeds into others. Furthermore, the continual repetition of the original loop (whose structure we can still discern despite its disintegration) is similar to the process by which the compression process continually attempts to impose some sort of uniformity on the chaos of sound waves in an attempt, however doomed, to master and control their heterogeneity (and thus the "meanings") inherent in sound. Thought of in these terms, the sounds of The Disintegration Loops are not infinitely plastic. The reappearance of the original loop, however dissolved its structure, becomes a sign of the power of sound that saturates the vibratory fabric as it works on the body. In this repetition we hear the forcible reiteration of this power and the histories implicated by that loop as they struggle to sonically emerge in various forms to mark the body. On the other hand, the disintegration process equally represents a sign of the ability of sound's alterity-its rarefactions - to resist this power and allow for the possibility of a number of different future resolutions of the sonic event with the listener (rather than imposed on the listener), because sound's heterogeneous disappearances open up the potential for other histories to emerge that can destabilize what appears to be the totalizing sonic unity of the original loop. Much like rarefactions existing in relation to the stronger forces of compressions, the disintegration process continues to dismantle the power of the original loop, insinuating itself into the functioning of that order over and over again over the course of a track, until the entire structure of the original loop's constellation of power-and therefore sound-has changed. ${ }^{20}$ So while the repetition of the original loop may force its own order and discourse, its histories, to re-emerge, the disintegration process differentially structures and sounds each loop by pushing forth a sonic mass of otherness-other discourses, other histories, other sounds-including disappearances, which represents the possibilities of different resolutions of the event, opening the door for the subject to invent other meanings and other discourses. In the loops, we can hear the continual appearance of possibilities to reconstitute the sonic event and the listening subject through the very otherness of their sonic disappearance, either as rarefactions or as disintegration, where even their not-sounds or not-power still persist as being inextricably linked to the larger discursive vibratory fabric; disappearance not only structures how the sound is heard, but how it could be heard. In this way, the loops come to represent a series of sonic singularities

20. Especially if one considers that the original loops represent a particular discursive unity and formality of Western canonical music in its metered rhythm, harmony and melody, localizing the power of this discourse within the boundaries of its one loop, and with it, its implicit notions of musical time and space. 
constantly re-opening to further articulation, related to but not statically defined by the other temporal spans of the other loops. However, the sonic warping of the disintegration makes these singularities "still inarticulate, too relaxed to be clear," the resolution of each loop not specified but "powering the music" while "focusing the next contraction, the next repetition." As such, the completion of each loop is forever but a partial resolution of the event, the loops' movement ensuring a constant reproduction of the event. As we have seen, the production of the sound, the subject and the resolution of the sonic event, whether in the vibratory fabric or in listening to music, are simultaneous to the extent that there is no final meaning of sound that pre-exists its doing or its subjects but is created only as it unfolds in conjunction with a listener who resolves these sonic singularities. In this sense, not only can the form/process of the loops serve as representing the operations of sound, speaking to the process of the sonic fragmentation of the subject, but listening to loops also initiates this fragmentation because it is sound, the continuous and shifting looping simultaneously multiplying the sonic events and possibilities while fragmenting the subject according to its own sounds and histories.

By actively engaging the loops, barriers between interiority and exteriority dissolve, the subject negotiates with its fluid, fragmented, decentered sounds and emerges itself as a dispersed sonic topography filled with the potential for future negotiations of sound and self. But at the same time, it also shapes subjectivity, through the processes described, according to complexifications of appearance and disappearance because The Disintegration Loops presents a complex and radically non-linear challenge to the temporality of appearance and disappearance. Basinski’s piece represents an exploration of the dynamic interplay of how time and history, appearance and disappearance, are active and integral forces that must be acknowledged when considering sound, and is done in such a way that the overlapping flow of random periods of both appearance (the repetition of the original loops) and disappearance (their disintegration) are not subject to a predetermined dialectical linearity but in fact trouble the "essence" of what sound is because both function simultaneously to influence the quality of heard sound. In the very unfolding of each loop, the decaying process problematizes the search for the essence of a self-contained centre of pure sound marked by strict temporal measures of appearance/disappearance. A track's everchanging structure acknowledges the very impossibility of this essence: each disintegrating loop, while a repetition of the same original loop, is differentially contextualized and temporalized in each unfolding, revealing its appearance and dispersing its disappearance in a different way, the music constantly emerging as a differential iteration of multiple and overlapping sonic events bleeding into each other and tainting the idea of the "pure" and unitary temporal sound event characterized 
by a strict dichotomization according to a coming to and a fading away. Further than this, being structured by the unfolding of the previous loop, the appearance of each loop is forever tied to its past, while at the same time, its own unfolding and disappearing structure structures the following unfolding loop and is thus forever tied to its own future; each loop is an instantiation of a dynamic and causal temporality and history marked simultaneously by both a loop's appearance and disappearance. Similarly, while sound can eventually be resolved in a sonic event with the listening subject, this event cannot have an independent existence marked by clear-cut temporal lines of "having appeared" or "having faded;" history always precedes it, informs it, shapes it, and then continues to live on following its inscription in the listening subject. Sound is literally a "coming into being" - it penetrates our bodies, our selves, our identities-and while sound's fading is this physicality literally draining away or "disappearing from being," it does so only after its own histories of appearances and disappearances have appeared in the body in such a way that the fluidity of the process which characterizes the constitution of the sonic event leaves the possibility that these appeared histories could once again disappear if reconstituted by the listening subject. In this sense, neither music nor sound are placed above or beyond the forces of history, in an empty temporality in which the pure essence of sound can exist independently and be strictly made to either appear or disappear. There is no such thing as a sound beyond history, or more specifically, a sound that is beyond the history of its overlapping and intertwined appearances and disappearances. The Disintegration Loops not only acknowledges this historical genealogy on numerous registers, where history in the form of the dynamism of decaying loops is its (un)structured principle, but incorporates this understanding into the very flows of the loops' appearances/disappearance which, much like the sonic process itself, dissolves their strict temporal dichotomization within the subject as he or she resolves these histories in a sonic event. 